\title{
Hispanismes
}

Revue de la Société des Hispanistes Français

$18 \mid 2021$

Murs, barrières, obstacles dans les mondes hispaniques II

\section{"J'écris ton nom sur les murs de ma ville ": notes sur les écritures exposées du documentaire chilien}

«Escribo tu nombre sobre los muros de mi ciudad»: apuntes sobre las escrituras expuestas del documental chileno

«I Write your Name on the Walls of my City»: Some Notes on the Exposed

Writings of Chilean Documentary

\section{Ignacio Albornoz Fariña}

\section{OpenEdition \\ Journals}

Édition électronique

URL : https://journals.openedition.org/hispanismes/14667

DOI : 10.4000/hispanismes. 14667

ISSN : 2270-0765

Éditeur

Société des Hispanistes Français

\section{Référence électronique}

Ignacio Albornoz Fariña, « « J'écris ton nom sur les murs de ma ville » : notes sur les écritures exposées du documentaire chilien », HispanismeS [En ligne], 18 | 2021, mis en ligne le 31 décembre 2021, consulté le 30 janvier 2022. URL : http://journals.openedition.org/hispanismes/14667 ; DOI : https://doi.org/10.4000/hispanismes.14667

Ce document a été généré automatiquement le 30 janvier 2022

\section{(c) $(1)$}

Les contenus de cette revue sont mis à disposition selon les termes de la Licence Creative Commons Attribution - Pas d'Utilisation Commerciale - Pas de Modification 4.0 International. 


\section{«J'écris ton nom sur les murs de ma ville » : notes sur les écritures exposées du documentaire chilien}

«Escribo tu nombre sobre los muros de mi ciudad»: apuntes sobre las escrituras expuestas del documental chileno

"I Write your Name on the Walls of my City»: Some Notes on the Exposed

Writings of Chilean Documentary

Ignacio Albornoz Fariña

Murals! They are living, breathing, seizing walls. And talking, wailing, murmuring walls. Murals! One cries out, the other doesn't. But these walls don't sell you anything. Agnès Varda, Mur murs (1981)

1 L'imaginaire du mur n'est pas étranger à la culture populaire chilienne des années 1970. «La muralla », se souvient-on, est le titre d'une des chansons les plus célèbres du groupe folklorique Quilapayún, composée sur un poème de Nicolás Guillén, dont l'œuvre lyrique a été d'ailleurs une riche source d'inspiration pour la musique populaire du continent. Dans son poème, Guillén plaide pour une communion des mains, chante la convergence d'un geste collectif. "Para hacer esta muralla tráiganme todas las manos ", écrit-il : " pour construire ce mur apportez-moi toutes les mains $»^{1}$. Art de son temps, le cinéma documentaire chilien des années 1970, pour l'essentiel encore « muet »- l'arrivée du son synchrone au Chili étant particulièrement tardive possède son propre répertoire de murs faits « de toutes mains ». Et celui qui prendra sa relève dix ans plus tard n'est pas en reste.

2 Il y a en premier lieu, dans ces cinémas, le mur-frontière ou le mur-clôture, celui qui détermine par exemple, dans des films aussi divers que Si todos los vecinos (1972) ou El sueldo de Chile (1971), le périmètre d'une propriété, l'étendue d'un domaine ; celui qui signale en somme les limites d'un enclos et qui sépare donc ce qui est dedans de ce qui 
- étranger, extrinsèque - reste dehors. Ce mur signale également la direction, le sens d'un regard, puisque c'est le plus souvent vers l'intérieur d'un lieu que l'objectif de la caméra porte son œil mécanique, comme le montrent les séquences initiales de Testimonio (1969) de Pedro Chaskel, où la caméra s'avance d'un pas lent vers trois patients d'un hôpital psychiatrique en ruines attendant derrière une porte à barreaux. Le mur-frontière, il va de soi, contribue à mettre en place toute une série d'oppositions structurantes, symptomatique qu'il est d'un état, et non pas d'un agissement: les possédants contre les "sans-parts", les filmeurs contre les filmés, les dépositaires légaux de la violence étatique contre ceux qui la subissent, et ainsi de suite.

Mais l'on reconnaît également, bien qu'en moindre quantité, des figures de ce que l'on pourrait appeler peut-être le mur-bouclier, celui qui, tout en dressant une frontière entre le dedans et le dehors, protège et secourt en quelque sorte ceux qui choisissent ne serait-ce qu'un instant de trouver en lui un abri, un rempart. Ce mur derrière lequel on peut trouver refuge n'est pas le fait d'un état, d'un objet donné ; il résulte plutôt d'un ensemble d'agissements: on le crée, on l'érige en l'utilisant, et c'est de là que lui viennent ses forces figurales. C'est, par exemple, l'arbre derrière lequel se barricadent un instant les manifestants les plus audacieux au début de Rebelión ahora (1983) de Rodrigo Gonçalves ou bien le bouclier que forment les barricades filmées par Gonzalo Justiniano dans Andrés de La Victoria (1985); mais il faudrait ranger aussi dans cette catégorie le mur "mouvant» que crée la ronde humaine de Somos + (1985) de Pedro Chaskel et Pablo Salas devant les canons à eau des chars des forces de l'ordre ou encore celui, tout aussi mobile, que tendent de bout à bout d'une ligne de cortège toutes ces banderoles portées en tête des manifestations de rue, et dont le plan qui clôt En nombre de Dios (1987) de Patricio Guzmán est un bon exemple.

Il est encore un troisième type de mur, à la lisière des deux autres, ni complètement frontière ni entièrement bouclier, mais un peu chacun d'eux à sa manière. C'est le mursurface, le mur-page, celui sur lequel on inscrit, on grave ou on colle un message, un slogan, une consigne ; en tout cas, celui sur lequel on écrit un texte que l'on adresse aux autres. Ce mur peut se rapprocher par moments du mur-frontière en ce sens qu'il implique un arrêt, une halte, puisqu'il enjoint le spectateur ou le passant à diriger son regard vers lui, à l'observer; mais il est en même temps proche du mur-bouclier en ce qu'il peut, comme lui, prendre forme à la faveur d'un agissement ou d'un geste. Il s'agit d'un mur « en acte ", qui ne devient ce qu'il est que lorsqu'une forme écrite s'impose à sa surface.

C'est sur les occurrences de ce type de mur que je voudrais m'attarder ici. Non pas pour en faire l'inventaire, mais pour tenter d'éclaircir par l'analyse - et sans trop m'occuper des chronologies - les contextes et les modalités sous lesquelles il apparaît. Deux moments particulièrement expressifs du cinéma documentaire chilien, entre lesquels se dresse de tout son poids l'intervalle traumatique du putsch militaire de 1973, retiendront mon attention: le documentaire de l'immédiate avant-dictature (1967-1973), et le documentaire «transnational $»^{2}$ qui, à partir de 1983, accompagne la lente reprise des forces démocratiques qui ont précipité la chute du régime vers la fin de la décennie. Quelques notions de Charles Pietri, Béatrice Fraenkel, Philippe Artières et Pedro Araya serviront d'appui à mes propos.

6 Qu'il s'agisse de petites phrases cocasses ou d'exclamations enflammées, le cinéma chilien a fait de ces écritures de rue, inscrites le long d'un mur ou d'une pancarte, un enjeu d'envergure, les déclinant selon diverses combinaisons. La récurrence du motif 
est telle que l'on peut aisément dire qu'il existe, dans ces cinémas, une véritable " fièvre scripturaire », tenant non seulement au moment même de l'écriture (lequel est d'ailleurs figuré très concrètement à l'écran) mais aussi à sa monstration, à sa mise en avant (par des mouvements de caméra ou des gestes d'emphase des intervenants euxmêmes). À regarder le documentaire de l'avant et l'après-putsch avec quelque attention, l'on remarque que l'écrit, dans ses divers avatars, en parasite avec ténacité l'image. Il parvient toujours à se faufiler, à se faire une place, çà et là, dans tous les coins imaginables : derrière la tête d'un témoin qui s'exprime de front, face à la caméra, devant celle d'un manifestant qui s'entête à être encadré par l'appareil, ou même tout en bas du cadre, sous forme de sous-titres incrustés. Mais tenons-nous-en, ici, à une seule de ces formes d'actualisation de l'écrit : celle des écritures exposées, les graffiti, les tags ou les fresques politiques que des mains souvent anonymes inscrivent sur les murs des villes.

7 Si besoin est de définition, reprenons celle - fort générique et issue de l'épigraphie savante - qu'en donne Charles Pietri, pour qui les graffiti «notent un message individuel et spontané, généralement dicté par la conjoncture du moment, noté avec un instrument de fortune [...] sur un support occasionnel et inhabituel, en utilisant l'écriture courante $"^{3}$. Instances d'une " écriture ordinaire $»^{4}$, les graffiti peuvent être englobés à grands traits dans l'univers des «écritures exposées» (displayed ou visible writings en anglais), telles que les comprend Béatrice Fraenkel, via Petrucci, Detienne et Corbier. Pour Fraenkel, les écritures exposées se définissent selon trois critères: la lisibilité, la visibilité et la publicité, lesquels "privilégient tour à tour l'écriture ellemême, le dispositif de sa diffusion et enfin sa réception $»^{5}$. En vue des récentes transformations des pratiques d'inscription, Fraenkel propose néanmoins d'ajouter à ces critères un quatrième aspect : leur performativité. Par là, Fraenkel entend désigner la capacité des écritures exposées d'organiser «la présence physique des personnes dans une modalité qui renforce leur être-ensemble $»^{6}$.

Cette dernière précision est cruciale, car les écritures exposées que je cherche à caractériser ici relèvent d'un contexte tout particulier : celui des manifestations de rue, des situations "de révolte». Elles sont à ce titre sujettes à une forme tenace d'évanescence, vouées par principe à la disparition, puisque "un écrit exposé en situation de révolte, écrit Pedro Araya, n'apparait que pour disparaitre $»^{7}$. En ce qui concerne le cinéma, l'évanescence est d'autre part un risque avec lequel le film militant doit souvent composer, comme le remarquait récemment Nicole Brenez : « les cinéastes qui dans la clandestinité tournent des images interdites savent bien qu'elles aussi risquent de disparaître au front ${ }^{8}$. Dans de telles situations, on le voit, l'écrit exposé révèle toute sa nature de fait situé, concret, vecteur d'activation de connaissances et d'expériences préalables. Car, renchérit Araya, "il n'y a point d'innocence du regard [...]. Il y a un savoir préexistant à tout abord [...] mis en tension devant ce qui apparaît $»^{9}$.

9 Puisqu'il s'agit après tout de murs, commençons par la première aventure cinématographique entreprise par Carmen Castillo, aux côtés de Pierre Devert et Fabienne Servan-Schreiber, dont le titre s'impose avec la force de l'évidence : Les murs de Santiago. Réalisé en 1983, le film se donne pour but de montrer, en agençant diverses voix et régions, la nouvelle physionomie du pays après presque dix ans de hiatus dictatorial. Au tout début, un plan s'attarde sur les digues du fleuve Mapocho, la principale artère fluviale de Santiago, filmées du côté du Pont de l'Archevêque au moyen d'un panoramique vers la droite. Le mouvement, assez discret, laisse alors 
découvrir une vieille fresque à demi-effacée. La voix profonde et songeuse de Castillo envahit aussitôt le champ sonore et se livre à un monologue où il est question précisément d'évanescence et d'inscriptions éphémères, mais aussi de persistance : «Je ne savais pas, dit-elle, qu'ils n'avaient pas pu, eux, effacer les traces de l'autre histoire sous le Pont de l'Archevêque, près de la gare. Non, je ne savais pas qu'il y a encore des empreintes et des mégots dans les murs de Santiago ».

C'est sur cette même fresque que s'arrête d'ailleurs Así golpea la represión, le documentaire qu'avaient réalisé en 1982, pour la télévision suédoise, Peter Nestler et Rodrigo Gonçalves. Le film commence par une séquence composée de vues fixes et mouvantes de Santiago, au gré d'une musique folklorique pleine de gaîté et d'allégresse. Le paysage somme toute touristique et pittoresque qu'offrent ces vues alterne par à-coups avec des plans montrant des lieux étroitement liés à la mémoire du coup d'État (le Stade National, camp de détention et torture, le palais présidentiel de La Monnaie). Au bout de la séquence, toutefois, la caméra finit par s'attarder sur les digues du Mapocho, qu'elle filme une fois de plus en effectuant un panoramique vers la droite, à contre-courant du fleuve. Çà et là, à la faveur de la lumière, des bouts de la vieille fresque révolutionnaire peuvent être aperçus avec précision : un groupe de mineurs, casque sur la tête, deux charrettes à bras, l'une portant du foin, l'autre des cailloux, un poing levé, le contour d'un vélo et, dans l'une des extrémités, le visage de Salvador Allende, en demi-profil (figs. 1 et 2).

Así golpea la represión

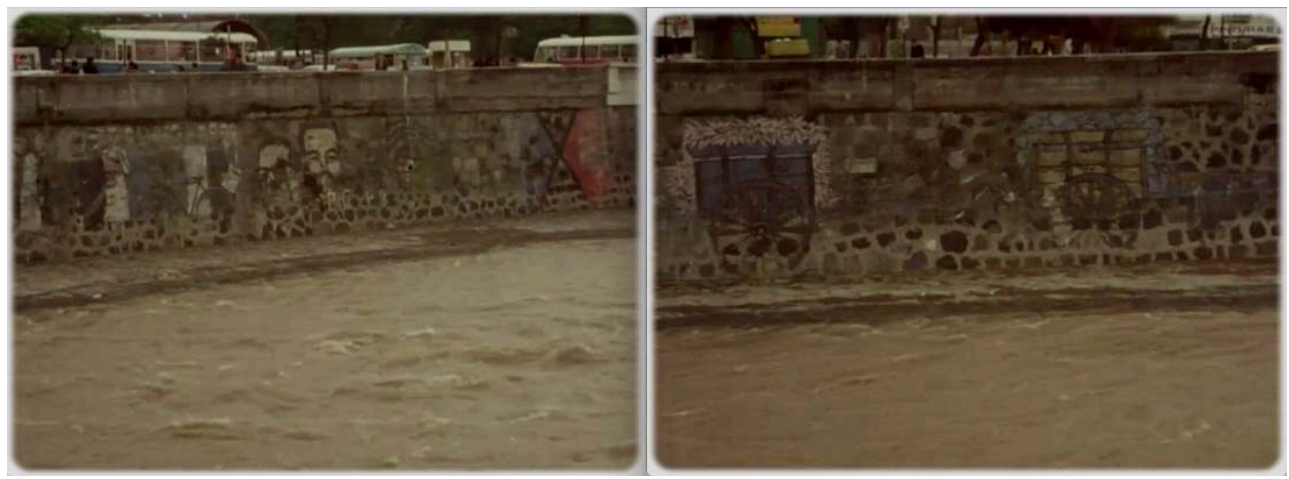

Peter Nestler et Rodrigo Gonçalves, 1982

Des fresques, il y en a aussi dans En nombre de Dios de Patricio Guzmán, film urgent, tourné dans le vif du très long cycle de manifestations qui secouèrent le régime entre 1983 et 1987. Le procédé par lequel Guzmán les donne à voir n'est pas dépourvu d'intérêt : le réalisateur les filme de front, à distance moyenne, au cours d'une séquence de la moitié du film qui suit l'intervention du théologien Ronaldo Muñoz. Il y en a, au total, cinq : elles célèbrent toutes le rôle des femmes dans le combat contre le régime. L'originalité de Guzmán - ou de ses opérateurs - consiste en l'occurrence à cadrer ces fresques de telle manière que les passants demeurent visibles à l'avant-plan. Ainsi, par un simple geste de montage, ce que l'on pourrait prendre prima facie pour une image fixe nous est révélé au bout de quelques secondes dans sa nature d'image mouvante, une fois que les passants font irruption dans le cadre, le traversant d'une extrémité à l'autre. Ce qui semble se manifester ici, en des mots de Camille Bui, est la «présence vivante, matérielle $»^{10}$ d'un ensemble de corps en action. Leur démarche - mouvement somme toute anodin - indique qu'on est en face de manifestations artistiques en temps 
présent ; des expressions en acte, contemporaines, qui interagissent avec leur milieu au quotidien.

Les fresques, ainsi, sont montrées en tant qu'éléments constitutifs d'un paysage urbain et social qui se reconstitue peu à peu, au rythme d'un quotidien parfois pesant. C'est d'ailleurs ce qu'un groupe de pobladoras, interviewé par Andrés Lübbert dans Chile, la cultura necesaria (1986) permet très concrètement de comprendre dans une séquence de la moitié du film. Interrogées par le réalisateur à propos du futur d'une fresque récemment effacée par des agents de la mairie, trois femmes du quartier déclarent, d'un air naturel et décontracté : «Sous peu, nous la repeindrons [...]. Quand nous avons besoin d'un mur, nous repérons ceux qui sont en bon état, nous les lavons et nous les peignons ». Lübbert insère ce bref entretien entre deux séquences dans lesquelles il fait défiler à l'écran une série de cinq ou six fresques de rue, avec pour fond, toujours, le passage des piétons (figs. 3 et 4), certains desquels n'hésitent pas d'ailleurs à regarder l'objectif de la caméra. Le témoignage de ces femmes, filmées en légère contre-plongée devant la fresque en question, acquiert de la sorte toute sa puissance d'actualité.

Chile, la cultura necesaria
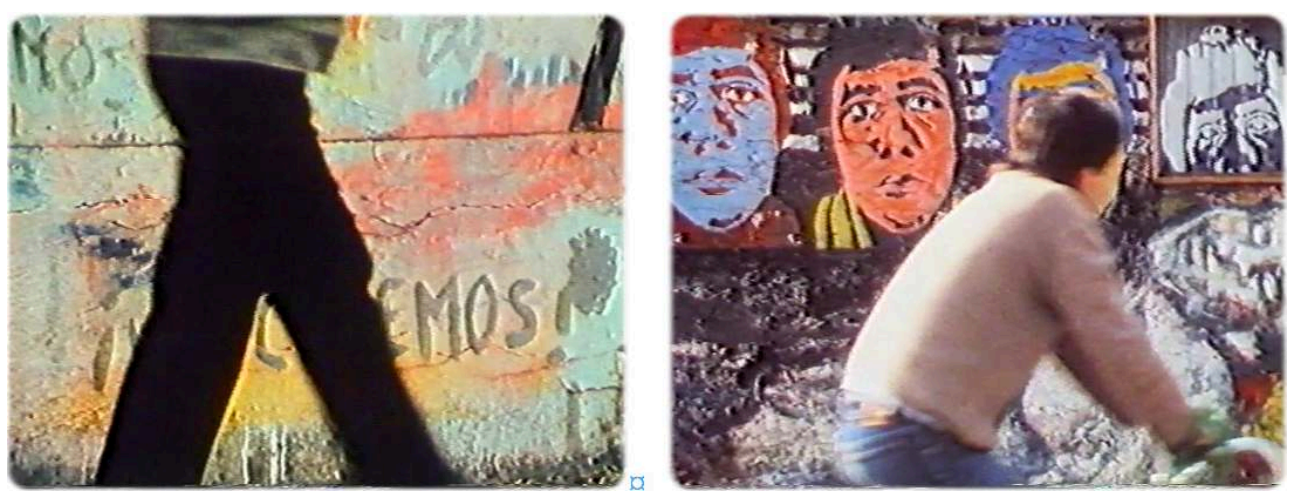

Orlando Lübbert, 1982

Dans le cadre du documentaire de l'avant-dictature, en revanche, les films qui représentent le mieux, d'un point de vue programmatique et officiel, la volonté de rassembler des formes d'expression artistique diverses dans l'espace de la ville sont Brigada Ramona Parra (1970), d'Álvaro Ramírez et Pintando con el pueblo (1971) de Leonardo Céspedes, tous deux réalisés sous l'égide du Centre de Cinéma Expérimental de l'Université du Chili (figs. 5 et 6). Dans ces deux œuvres, en effet, le motif du graffiti - ou plutôt des fresques de rue - est convoqué de manière assez tangible, à travers deux campagnes populaires d'intervention dans l'espace public cherchant d'abord à appuyer la candidature de Salvador Allende puis à célébrer sa victoire. 
Brigada Ramona Parra et Pintando con el pueblo
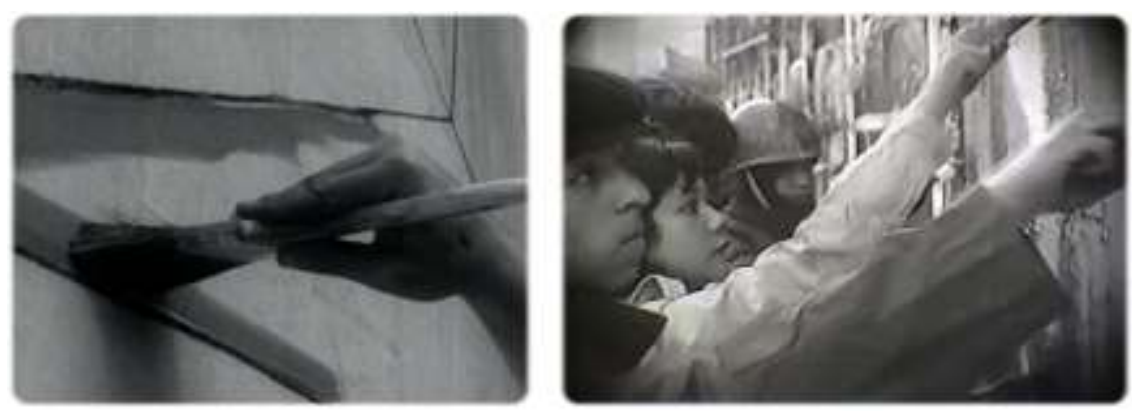

Álvaro Ramírez, 1970 et Leonardo Céspedes, 1971 plus intéressante les agissements des citoyens sur les murs de la ville. Tourné en 1970, à la veille de la victoire de la coalition de Salvador Allende dans les urnes, Venceremos (1970) est certainement l'une des œuvres cinématographiques les plus emblématiques de la période de l'Unité Populaire. Réalisé par Pedro Chaskel et Héctor Ríos, ce moyenmétrage d'une vingtaine de minutes agence à dessein des images se contredisant les unes les autres. Outre ce montage dialectique opérant par contrastes, les cinéastes présentent une bande-son composée majoritairement de chansons populaires issues du répertoire du folklore chilien ou de la variété européenne. Il en résulte un film cru, viscéral, quoique par moments quelque peu manichéen, dans lequel sont dits tous les fléaux d'une inégalité brûlante, séculaire.

La synthèse y est atteinte lorsque le plan d'un mur, sur lequel on peut très nettement lire, peint à la brosse, en noir, le mot «Basta » (fig. 7), nous est montré, alors que le film atteint son paroxysme dramatique : « ça suffit », s'exclament les réalisateurs, après une séquence particulièrement prégnante dépeignant des émeutes, des affrontements avec les forces de l'ordre et des faits de violence policière, laquelle est suivie par un écran noir. C'est par un fondu enchaîné que le mot surgit des ténèbres. Le plan, quant à lui, est fixe ; la caméra y est située à hauteur de vue, elle prend le muret de front, le laissant investir tout l'espace du cadre. Ce mur total, contre lequel le film se heurte dans son déroulement, stoppe d'un même élan le défilement des images et le mouvement de la bande-son, suspendant pour quelques instants le récit et annulant par là même le jeu progressif de contrastes qui l'animait depuis le début. Ce qui suit est une série d'images de liesse, prises sur le vif, dans les rues, parmi des groupes de manifestants extatiques, qui jettent parfois des regards pleins d'émotion dans l'objectif de la caméra: l'Unité Populaire vient de remporter l'élection présidentielle. 


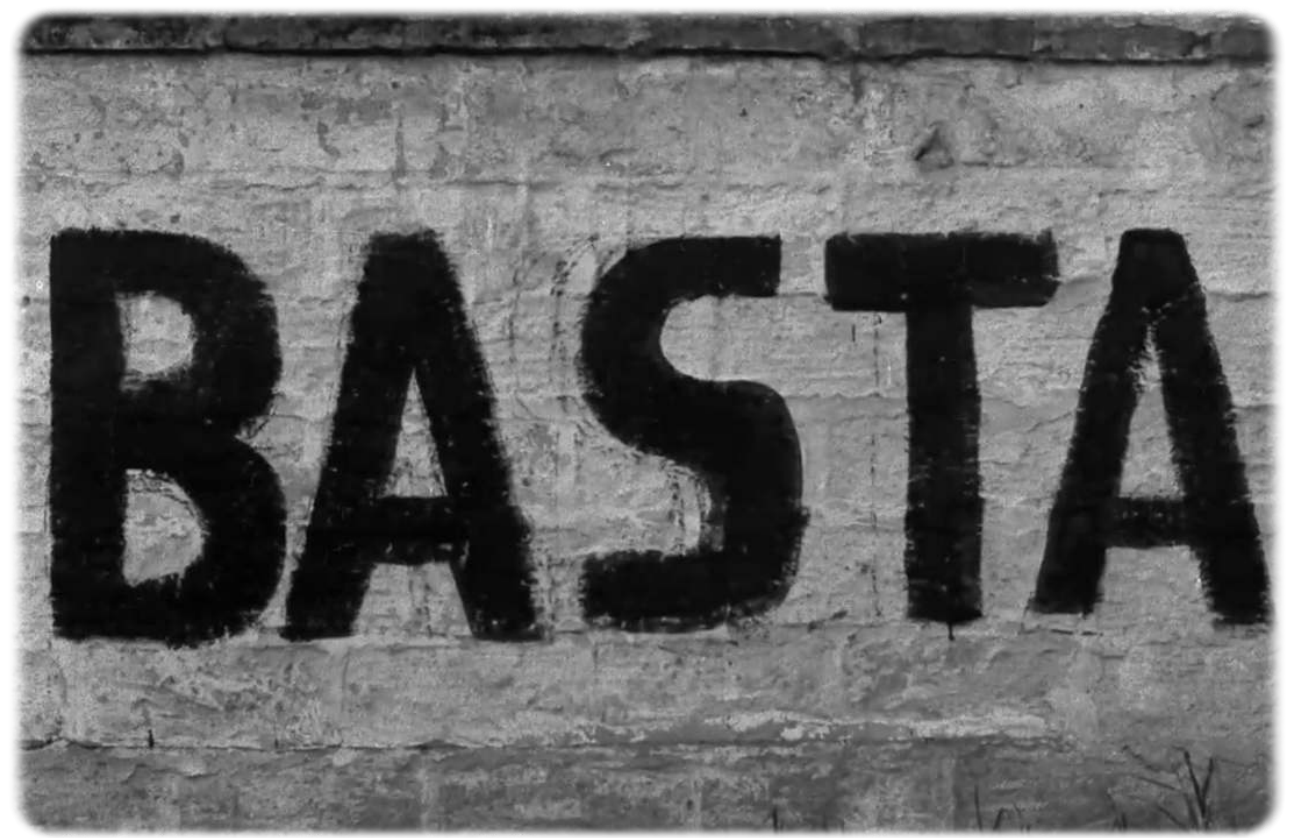

Pedro Chaskel et Héctor Ríos, 1970

Quatre scènes du cinéma de la reprise démocratique viennent ici à l'esprit: des manifestants, une bombe de peinture en main, écrivent des tags politiques sur des murs. Ils annoncent la date de la prochaine manifestation, ils esquissent à la hâte un slogan, un dessin à fort contenu politique. L'écriture est donnée à voir dans sa genèse : au moyen de plans souvent très brefs, l'on assiste ainsi à la naissance d'un mot, d'une phrase, à sa réalisation en temps réel, avec pour surface d'inscription, toujours, un mur. L'on songe par exemple à ce jeune homme qui, dans En nombre de Dios, s'agenouille furtivement pour écrire à l'aérosol, sur un mur mitoyen, la phrase : «l'espoir chrétien est socialiste ", qu'il trace d'ailleurs brusquement, toujours prêt à déguerpir. Ou bien à cette jeune femme qui, vers la fin de Chili, je n'invoque pas ton nom en vain, film du collectif Cine-Ojo tourné dans la clandestinité, écrit sur la pointe des pieds la consigne " vers la grève ", et qui traverse ensuite le cadre, au milieu d'une foule qui s'avance en direction de la caméra, dans un plan tout aussi furtif (fig. 8). 
Chile, no invoco tu nombre en vano

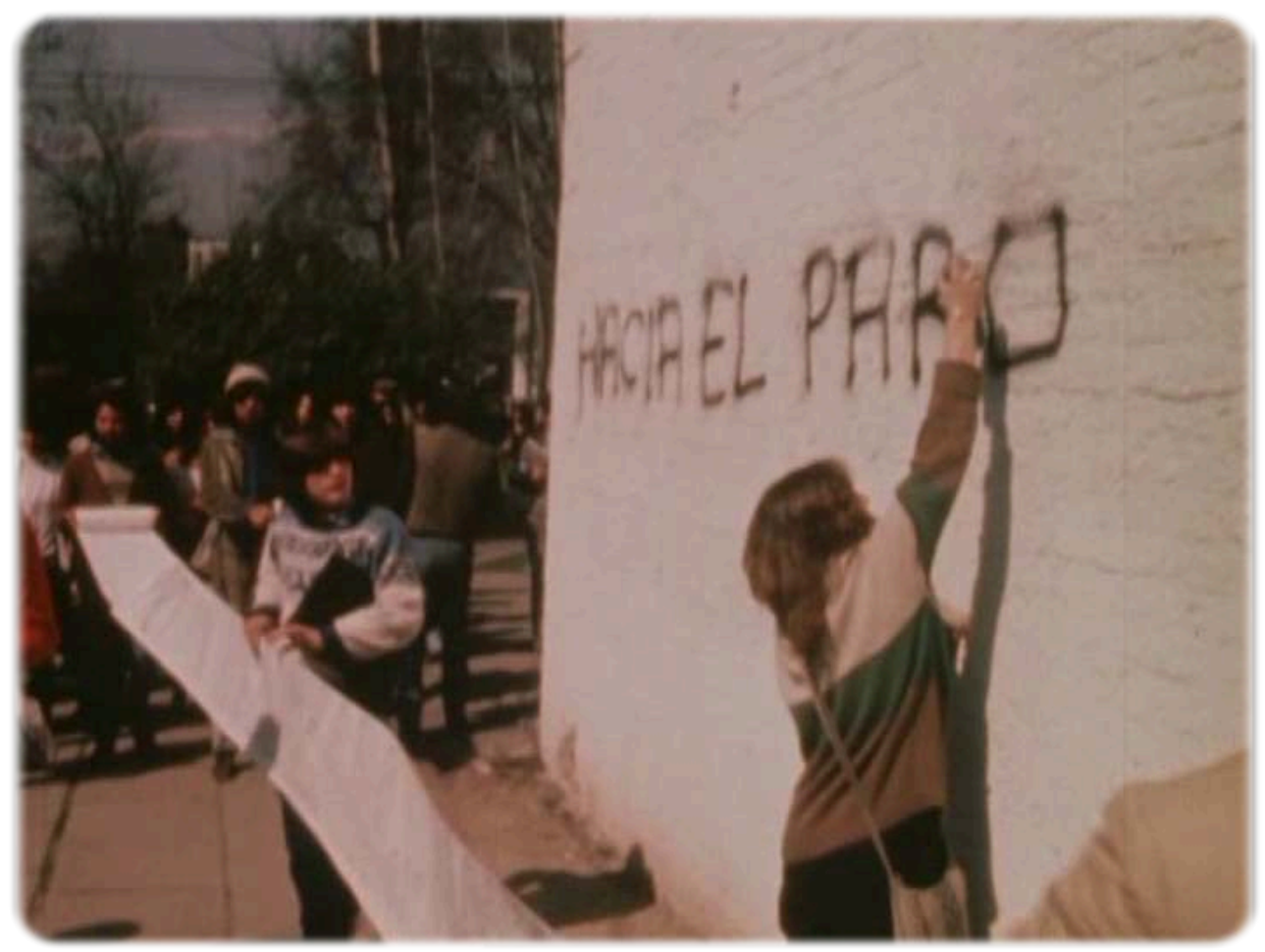

Colectivo Cine-ojo, 1983

Ce type d'écriture "en devenir ", montrée au moment même de sa genèse, revient assez souvent dans les documentaires de cette période. Rodrigo Gonçalves en fait même - et cela n'est point anodin - le titre de Rebelión ahora, film qu'il tourna au Chili en 1983. En effet, l'un des premiers plans de ce documentaire montre, par l'intermédiaire d'un zoom-arrière, et juste après une courte introduction à l'image frémissante où l'on voyait des confrontations entre les forces de l'ordre et les manifestants, un jeune homme qui peint encore une fois sur un mur, muni d'une bombe de peinture. Son message, que l'on découvre seulement lorsque la caméra complète son mouvement de retrait, est simple, sec, catégorique et se détache nettement du muret en parpaings qui l'accueille : « Rebelión ahora ». Les mots sont d'un rouge vif, presque saignant (fig. 9).

Le geste se reproduit à nouveau dans Dulce Patria de Andrés Racz (1985), où en pleine nuit un homme cagoulé trace sur le mur d'un quartier populaire, à la dérobée, un poing tendu, fermé, tenant une sorte de faux (fig. 10). Le plan, de très courte durée et pauvrement éclairé, est en outre pris de dos. Chela (1986) en fournit une autre variation. Ce remarquable reportage de la télévision suédoise concentré sur le quotidien d'une jeune habitante d'un quartier populaire de Santiago met en scène plusieurs instances d'écriture exposée. Dans l'une d'entre elles, un groupe de trois adolescentes, parmi lesquelles figure naturellement la protagoniste, peint à la brosse le seul mot «creyeron». C'est le plan suivant qui donnera la clé de l'énigmatique inscription: sur un mur extérieur d'aspect rudimentaire, le message jouxte en l'occurrence le visage de Salvador Allende, peint au pochoir. 
Rebelión ahora

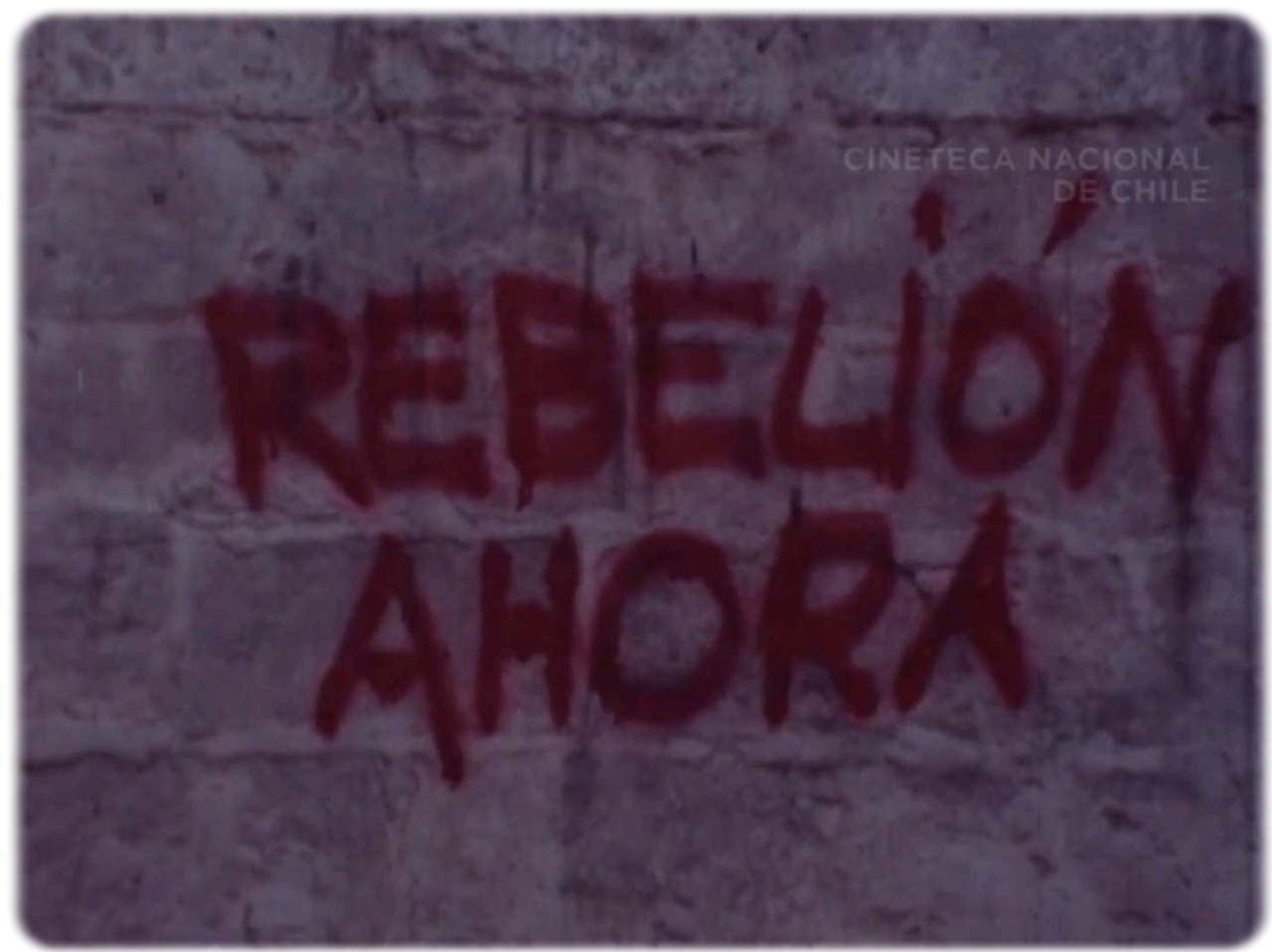

Rodrigo Gonçalves, 1983

\section{Dulce patria}

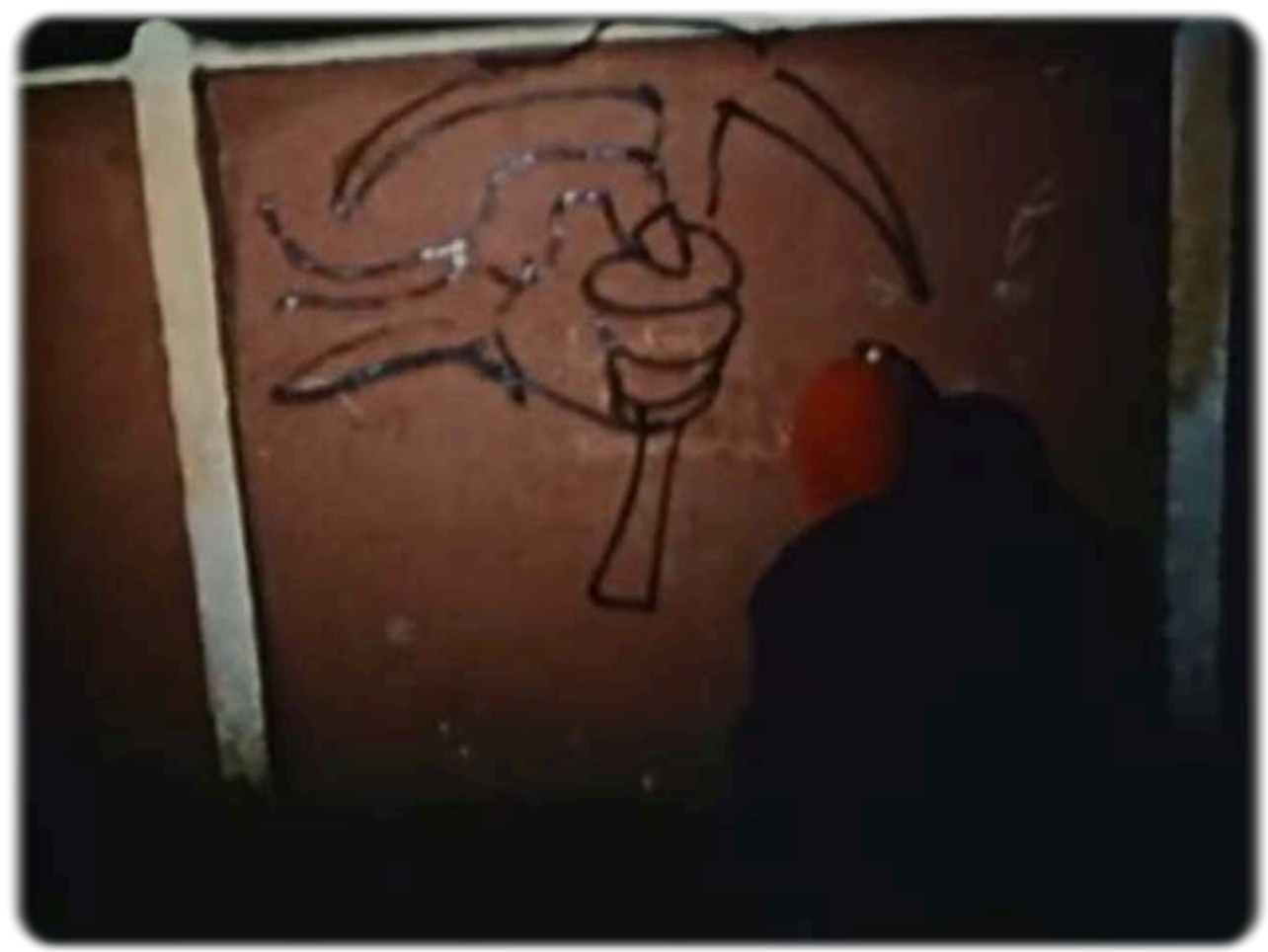

Andrés Racz, 1985 
Donnant corps à une vision de la ville en tant que surface susceptible d'être appropriée et resignifiée par l'écriture et le dessin, les plans «muraux » que j'ai évoqués jusqu'ici sont doublement éphémères (car non seulement ils sont très brefs, mais ils fixent des objets qui sont eux-mêmes provisoires, passagers). En dirigeant donc l'objectif de leurs appareils vers les murs peints d'une ville « en dispute " pour les enregistrer ne serait-ce qu'un instant, les cinéastes produisent l'événement. Car ils octroient d'une part une présence temporelle à l'éphémère, nous rappelant, avec Artières, que « la photographie permet à l'écriture éphémère de ne plus l'être $\aleph^{11}$. Et, de l'autre, car ils aident à instituer par la prise de vue un véritable événement d'écriture, au sens où Fraenkel entend ce concept, c'est-à-dire comme "la transformation d'un fait d'écriture publique et primaire, appartenant à un répertoire connu d'actions, en acte singulier, mémorable $»^{12}$.

Vecteur d'actes mémorables, le mur apparaît ainsi, dans les deux cycles documentaires commentés, comme un lieu fécond où, pour reprendre des mots de Camilo Trumper, se croisent «ville, production visuelle et pratiques de citoyenneté politique enracinées dans l'espace public $»^{13}$. Dans le cinéma de l'avant-dictature, le mur permettait en effet de conjurer par la lettre, avec un grand sens de l'économie expressive, le mutisme auquel les limitations et contraintes technico-sonores des appareils de filmage circonscrivaient les sujets à l'époque : le mur, ainsi, "parlait " là où la voix faisait défaut. Dans le cadre du cinéma mobilisé de la reprise démocratique, en revanche, le mur devient l'outil d'une véritable esthétique de l'urgence, conjuguée au présent par des sujets déjà doués de parole : tantôt il exhibe les traces d'un passé révolu, tantôt il est investi, empli et recouvert en temps réel, sous nos yeux. À dix ans d'écart, l'un et l'autre rappellent en somme que toute écriture, aussi banale ou éphémère qu'elle soit, suppose un geste qui lui donne vie.

\section{BIBLIOGRAPHIE}

Pedro ARAYA, « Lo que aparece, lo que desaparece », Antonio Castillo Gómez (éd.), Culturas del escrito. Del Renacimiento a la contemporaneidad, Madrid, Colección de la Casa de Velázquez, 2015, p. 45-63.

Philippe ARTIÈRES, Maëlle BAZIN \& Frédéric LAMBERT, « Écritures éphémères, écritures fragmentaires, écritures ordinaires », Communication \& Langages, nº 197 (2018), p. 111-124.

Nicole BRENEZ, Manifestations : écrits politiques sur le cinéma et autres arts filmiques, Réville, De l'incidence, 2019.

Camille BUI, Cinépratiques de la ville : documentaire et urbanité après Chronique d'un été, Aix-enProvence, Presses Universitaires de Provence, 2018.

Daniel Fabre, Les écritures ordinaires, Paris, P.O.L, 1993.

Béatrice FRAENKEL, « Les écritures exposées », Linx, nº 31 (1994), p. 99-110. 
Béatrice FRAENKEL, « Catastrophe Writings: In the Wake of September 11 », Marija Dalbello et Mary Shaw (éds.), Visible Writings: Cultures, Forms, Readings, New Jersey, Rutgers University Press, 2011, p. 305-318.

Béatrice FRAENKEL, « La notion d'événement d'écriture », Communication \& Langages, nº 197 (2018), p. 35-52.

José Miguel PaLAcios, « Chilean Exile Cinema and its Homecoming Documentaries », Rebecca Prime (éd.) Cinematic Homecomings: Exile and Return in Transnational Cinema, New York, Bloomsbury, 2016, p. 147-167.

Charles PIETRI, « Graffiti », dans Christiana respublica. Éléments d'une enquête sur le christianisme antique, Rome, École Française de Rome, 1997, nº 234, p. 1469-1490.

Camilo TRUMPER, Ephemeral Histories. Public Art, Politics, and the Struggle for the Streets in Chile, Oakland, University of California Press, 2016.

Filmographie

Andrés de La Victoria, Gonzalo Justiniano, 1985.

Así golpea la represión, Peter Nestler et Rodrigo Gonçalves, 1982.

Brigada Ramona Parra, Álvaro Ramírez, 1970.

Chile, la cultura necesaria, Orlando Lübbert, 1982.

Chile, no invoco tu nombre en vano, Colectivo Cine-ojo, 1983.

Dulce patria, Andrés Racz, 1985.

En nombre de Dios, Patricio Guzmán, 1987.

Pintando con el pueblo, Leonardo Céspedes, 1971

Rebelión ahora, Rodrigo Gonçalves, 1983.

Somos +, Pedro Chaskel et Pablo Salas, 1985.

Testimonio, Pedro Chaskel, 1969.

Venceremos, Pedro Chaskel et Héctor Ríos, 1970.

\section{NOTES}

1. Cf. Nicolás GUILLÉN, « La muralla », Summa poética. «La paloma del vuelo popular », selección de Luis Iñigo Madrigal, Madrid, Cátedra, 1997, p. 163.

2. José Miguel PALACios, «Chilean Exile Cinema and its Homecoming Documentaries ", Rebecca Prime (éd.) Cinematic Homecomings: Exile and Return in Transnational Cinema, New York, Bloomsbury, 2016, p. 147-167.

3. Charles PIETRI, « Graffiti », Christiana respublica. Éléments d'une enquête sur le christianisme antique, Rome, École Française de Rome, 1997, nº 234, p. 1469-1490.

4. Cf., sur ce point : Daniel Fabre, Les écritures ordinaires, Paris, P.O.L., 1993.

5. Béatrice FRAENKEL, « Les écritures exposées ", Linx, nº 31 (1994), p. 99-110, p. 102.

6. Béatrice FRAENKEL, "Catastrophe Writings: In the Wake of September 11 ", Marija Dalbello et Mary Shaw (éds.), Visible Writings: Cultures, Forms, Readings, New Jersey, Rutgers University Press, 2011, p. 305-318, p. 316. 
7. Pedro ARAYA, «Lo que aparece, lo que desaparece ", Antonio Castillo Gómez (éd.), Culturas del escrito. Del Renacimiento a la contemporaneidad, Madrid, Colección de la Casa de Velázquez, 2015, p. $45-63$, p. 60.

8. Nicole BRENEZ, Manifestations : écrits politiques sur le cinéma et autres arts filmiques, Réville, De l'incidence, 2019, p. 6.

9. Pedro ARAYA, « Lo que aparece, lo que desaparece », op. cit., p. 48.

10. Camille BUI, Cinépratiques de la ville: documentaire et urbanité après Chronique d'un été, Aix-enProvence, Presses Universitaires de Provence, 2018, p. 111.

11. Philippe ARTIÈRES, Maëlle BAZIN \& Frédéric LAMBERT, «Écritures éphémères, écritures fragmentaires, écritures ordinaires », Communication \& Langages, no 197 (2018), p. 111-124, p. 118.

12. Béatrice FRAENKEL, "La notion d'événement d'écriture ", dans Communication \& Langages, nº 197, 2018, p. 35-52, p. 45.

13. Camilo TRUMPER, Ephemeral Histories. Public Art, Politics, and the Struggle for the Streets in Chile, Oakland, University of California Press, 2016, p. 160.

\section{RÉSUMÉS}

À la faveur d'une approche éminemment essayistique et non-linéaire, le présent texte cherche à dresser un aperçu des différentes instances d'écriture exposée que découvre sur les murs de Santiago le documentaire chilien; ce, dans deux moments particulièrement expressifs de son histoire récente, entre lesquels se dresse l'intervalle traumatique du putsch militaire de 1973 : le documentaire de l'immédiate avant-dictature (1967-1973), et le documentaire transnational qui, à partir de 1983, accompagne la lente reprise des forces démocratiques qui ont précipité la chute du régime vers la fin de la décennie.

Drawing on an eminently essayistic and non-linear approach, this text seeks to provide an overview of the different instances of displayed writing that Chilean documentary has encountered on the walls of Santiago, in two particularly expressive moments of its recent history, between which lies the traumatic interval of the 1973 military putsch: the documentary of the immediate pre-dictatorship (1967-1973), and the transnational documentary that, from 1983 onwards, accompanies the slow revival of the democratic forces that precipitated the fall of the regime towards the end of that decade.

\section{INDEX}

Mots-clés : documentaire, murs, écritures exposées, dictature, cinéma chilien

Keywords : documentary, walls, displayed writings, dictatorship, Chilean cinema

\section{AUTEUR}

\section{IGNACIO ALBORNOZ FARIÑA}

Paris VIII Vincennes-Saint-Denis 